\title{
INDEPENDENCE OF CHROMOSPHERIC ACTIVITY AND SOFT X-RAY FLARING ON THE FLARE STAR EV LACERTAE
}

\author{
C.W. Ambruster ${ }^{1}$, B.R. Pettersen ${ }^{2}$, S.L. Hawley ${ }^{3}$, L.A. Coleman, ${ }^{4}$ and S. Sciortino
}

1 Department of Astronomy, Villanova University, Villanova, PA 19085

${ }^{2}$ Institute of Theoretical Astrophysics, University of Oslo

3 Astronomy Department, University of Texas, and McDonald Observatory

4 Dept. of Physics \& Astronomy, U, of Arkansas

${ }^{5}$ Osservatorio Astronomico di Palermo

\section{ABSTRACT}

EXOSAT observed the fare star EV Lacertae for 17 hr over 2 days in October 1985. Two flaring episodes were recorded. During a sicnificant fraction of these observations, IUE, photometric and spectroscopic coverage was available. A 2 -hour long period of $\mathrm{X}$-ray flaring was observed during which there wis no $U$ band activity and almost no chromospheric activity. On the other hand, two $\Delta U \sim 1{ }^{\circ} \mathrm{m}^{\circ} .5$ optical flares produced normal chromospheric enhancements, but only a weak X-ray response. We suggest that these and a few other observations of stellar flares may imply the occasional existence of magnetically isolated regions in $\mathrm{M}$-dwarf atmospheres.

\section{INTRODUCTION}

EV Lacertae is a classical flare star (dM4.5e) with a rather normal rotation period of 4.375 days (Pettersen, Kern, and Evans 1983), which nevertheless showed unusually violent X-ray flaring in HEAO-1 All Sky Survey data $(0.5-20 \mathrm{keV})$ : 2 flares, both with a faclor 50 flux increase and durations of 3-4 hours were seen within 5 days (A mbruster, Snyder and Wood 1981). Optical 'superflares' where $\Delta U \sim 5$ mag have also been observeci (Kodaira, Ichimura and Nishinura 1976; Roizman and Shevchenko 1982). In order to searcls for clues to the unusual flaring activity of this star, multi-wavelength observations involving EXOSAT, IUE, ground-based optical spectroscopy, and UBVR photometry were obtained on 13 October 1985 and 14 - 15 October 1985.

\section{OBSERVATIONS}

'The EXOSA'T observations on both days were made simultancously with the LE (0.04-2 keV) and ML $(2-6 \mathrm{keV})$ detectors. EV Lac was observed for $5 \mathrm{hir}$ on 13 October 1985, and for 12 hir on 14-15 October 1985.

The IUE observations were part of an extended program in which the star was observed 4 hours each day for 9 consecutive days between 7 and 15 October 1985; a partial report is given in Ambruster et al. (1986), and a larger paper is in preparation. On each day, alternating low dispersion LWP $(2000-3000 \AA)$ and SWP $(1200-2000 \AA$ ) exposures were obtained, resulting typically in 2 exposures in each camera per night. The line strengths were measured using a routine of $F$. Walter w !ich fits a quadratic background and a Gaussian of 6 AFWIIM, the instrumental resolution, to the lines

Optical spectroscopy was done with the Intensified Dissector Scanner Spectrograph on the $2.7 \mathrm{~m}$ telescope at McDonald Observatory. The spectral coverage extended from $3600-6600 \AA$ with an effective spectral resolution of approximately $6 \AA$. The individual spectra, with exposure times between $15 \mathrm{sec}$ and $1 \mathrm{~min}$, were co-added over the duration of each IUE exposure.

Eight filter photometry was obtained on the $0.9 \mathrm{~m}$ telescope at McDonald observatory: U, B, V, R, I $\alpha$ wide and narrow, Stromgren $\mathrm{H} \beta$ wide and narrow). Integration times were $1 \mathrm{sec}$, time resolution was 9 sec (the time to cycle through all filters plus $1 \mathrm{sec}$ for filter whecl rotation). 


\section{RESULTS}

While no X-ray 'super-flares' were seen prolonged, and apparently almost entircly coronal, X-ray flaring activity was seen during a $\sim 2$ hour period on 13 October. A single X-ray flare, also lasting 2 hours, was seen on 15 October.

\section{October 1988: Extended X-Ray Flaring}

EXOSAT obstrvations of EV Lac began al 0410 UT during a brief gap in U-band coverage because of clonds. The start of the extended X-ray activity began $10 \mathrm{~min}$ later at $0.150 \mathrm{UT}$ with a sharp rise in the count ratte. 'l'he X-ray light curve (Fig. 1) suggests multiple events during the next 2 hours (0440 0040 U'l'), for example, near $0450 \mathrm{U} \mathrm{I}^{\prime}, 0550 \mathrm{U}$ 'T, and $0620 \mathrm{UT}$. There are also marginal events near $0510 \mathrm{UT}$ and $0535 \mathrm{U}^{\mathrm{U}} \mathrm{T}$.

'The: low point at the start of the light curve at 0440 U'T may or may not belong to the pre-outburst phase: similar low points between 0550 and 0 bise UT are clearly only a brief interlude in the prolonged activity.

U-band coverage bregan again at $0457,7 \mathrm{~min}$ after the $X$-ray rise; there was no sign of activity for the nuxt hour. There is another bricf gap in coverage because of weather between 0512 and 0524 UT.

Spectroscopic coverage began slightly before U-band coverage at 0447 UT, $\sim 3$ min prior to the X-ray rise: 'The II $\beta$ data hetween 0447 and 0630 U'T on this night were co-added over 5 min intervals to search for responses to the $X$-ray activity. The equivalent widhs were normal at 0.447 and $0453 \mathrm{UT}$, high at 0458 U'T, and still sonewhat high but decreasing at $0503 \mathrm{U}$ T (Fig. 3). The average $\mathrm{H} \beta \mathrm{EW}$ for this $2 \mathrm{hr}$ period is typical of non-flare values between 7 and 15 October 1985, nevertheless, the high point at 04158 UT and the chear response to the $\Delta U=1^{m} .8$ flare 1 hr later are clearly real.

There wiss no IUli coverage prior to 0557 UT, 1 hour after the $X$-ray rise.

No impulsive phiase event was observed at the start of the X-ray activity because of lack of coverage. The only cunstraint that we can set is that there could have been no major U-band flare near the X-ray rise at 0150 U'I: such flares take many minules to decay and the decay phase would still have been visible in the U-band light curve which began 7 min later. Generally both the soft X-ray and $11 \beta$ cmissions represent the gradual fliase of a llare; if that is true here, there is consistency with the chromospheric evaporation model in that an increase is seen in coronal emission before a rise is seen in chromospheric emission.

The time between 0450 and $0548 \mathrm{UT}$ is clearly a time of considerable coronal activity, yet there is no associated photospheric (U-band) activity; nor, as mentioned above, could the $X$-ray activity have been precipitated hy some unusually large U-band impulsive event prior to the start of observations. Furthermore, except for the one unambiguous high point at $0458 \mathrm{UT}$, there is also no chromospheric (II $\beta$ ) response to this prolonged X-ray activily.

13 October 1985, Flare $\left(\Delta U=1^{\prime \prime} .8\right)$ at 0548 U'

Nore normal hare behavior wis seen in simultaneous observations of a strong flare at 05.48 UT when the U. bind light curve recorded a $1^{m} .8$ flare.

'The X-ray light curve (Fig. 1) shows a single high point at this time followed by 2 low points at the quiescent ferel; alllough soft $X$-ray flux is generally associated with the gradual plabe of a fare, in this case it secms possible that we have detected a soft $X$-ray impulsive event.

'The chromospleric layers responded to this flare in all observed lines: $\mathrm{H} \beta, \mathrm{Ca}$ II $\mathrm{K}$, and $\mathrm{Mg}$ II (2800 $X)$. The 116 liW's at 05.18 and 0553 UT were higher than at any time prior to 0630 UT; these 2 points were followed by a drop and then a smaller but extended enhancement at 0615 UT (Fig. 3). Co-added over the concurrent IUL exposure (LWP 6900L: 0557-0629 UT), the $11 \beta \mathrm{EW}$ shows a $2.6 \sigma$ enhancement. A stronger response was cvident in the Ca $11 \mathrm{k}$ FW's averaged over this sime time period, which show a $6 \sigma$ increase (crror bars are too large in 5 min co-added expesures for meaningful results). No Il (2800 $\AA$ ) was saturated in LWV (9900L: this occirred only twice in 17 I.WP spectra obtained over the 9 nights of the campaign, and in bulh cases significant U-band llares occurred during the exposure. 
There are no significant enhancements in the transition region C IV (1550 $\lambda$ ) line in 2 exposures on this night (SWP 26926L, SWP 26926L). However, the first SWP exposure begall almost 1 hour after the $1^{m} .8$ U-band flare at $0548 \mathrm{UT}$, and no response would be expected.

The only remaining activity on this night was a tiny $0^{m} .2 \mathrm{U}$-band flare at 0633 UT, about 13 min after the final X-ray rise of the night at $0620 \mathrm{UT}$; there is no noticeable response at other wavelengths.

There is, however, a probably significant $1 \sigma$ enhancement in the U-band continuum starting at about 0617 UT, at the start of the final decay in the X-ray light curve, suggesting some heating of the photosphere from above.

Finally, it is interesting that when the extended X-ray activity between 0410 and about 0720 is over, the quiescent level is quite constant: an analysis for quiescent, non-periodic variability using the method of Collura et al. (1987) shows no significant variability above $20 \%$ amplitude from 0720 to 1027 U'T.

\section{October 1985, X-Ray Flare at $0500 \mathrm{UT}$}

The EXOSAT light curve (Fig. 2) shows a sharp rise at $0500 \mathrm{UT}$, following $6.5 \mathrm{hr}$ of variable quiescent Rux (2216 $0410 \mathrm{UT}$ ). The variability was significant at the $99.9 \%$ level with a characteristic timescale > 1 hour (Collura et al. 1987; for an extensive application of this method to active cool stars, see $A$ mbruster, Sciortino and Golub 1987). Significant quiescent variability continued after the flare had subsided (0640 1001 UT) at a slightly lower, but still significant, level (95\%).

The pre-flare and flare count rates in the $\mathrm{ME}$ are the same within errors $(0.54 \mathrm{q} 0.06$ and 0.61 q 0.09 , respectively), so that this flare was quite soft: the combined $L F$ and ME data yield $\mathrm{k}^{\prime} \mathrm{T}=1.4 \mathrm{~g} 0.2 \mathrm{keV}$ (90(the distance of EV Lac is $\mathbf{5 p c}$ ). The quiescent spectrum is essentially the same, but less well determined: $\mathrm{k}^{\prime} \mathrm{T}=1.5 \mathrm{q} 0.2 \mathrm{keV}$, and chi-squared $\sim 2$. These temperatures agree with those found by Schmitt et al. (1987) for oller $\mathrm{M}$ dwarfs observed by EXOSAT.

U-band coverage began at $0.159,1$ min before the $X$-ray rise. $\Lambda$ small, $\Delta U=0^{\prime \prime} .4$ thare occurred at 0502 UT at, or slightly after the peak of the X-ray flare. A second small event $\Delta U=0^{m} .4$ ) was observed at 0601 UT, coincident with a small rise in the X-ray flare decay light curve.

Because there was no spectroscopic or IUE coverage till 0550 UT, 50 min after the start of the X-ray flare, no infornation on the chromospheric response to the main $X$-ray flare is available. During the first IUE exposure (LWP 6916L; 0550 - $0625 \mathrm{UT}$ ), however, the It $\beta \mathrm{EW}$ from spectra co-ilded over this interval is $2.6 \sigma$ high, most likely reflecting the $\Delta U=0^{m} .4$ thare which occurred at $0601 \mathrm{UT}$. P'robably for the same reason, the $\mathrm{Mg}$ Il $(2800 \AA)$ flux ir, marginally $(1 \%)$ high.

15 October 1988: Flare $\left(\Delta U=1^{\text {nn } .5) ~ a t ~} 0625\right.$ UT

An X-ray spike occurred simultaneously with this U-Land hare at 0625 UT, punctuating the last stages of the decay of the X-ray flare at 0500 UT. As with the $\Delta U=1^{m} .8$ flate at 0548 UT' on 13 October, this spikike seems to represent an impulsive soft $\mathrm{X}$-ray event.

Like the $\Delta U=1^{m} .8$ fare on 13 October, the chromospheric response is seen in both $11 \beta$ ( $6 \sigma$ increase)

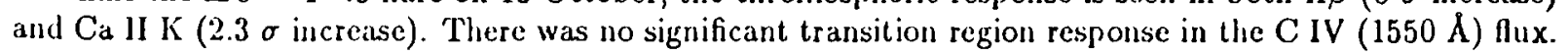

\section{DISCUSSION AND CONCLUSIONS}

We can summarize the results of the observations as follows:

- Fairly large U-band flares were associated with small, shlort duration X-ray spikes; significant X-ray flares wcre associated with small $\left(\Delta \mathrm{U}=0^{\circ} \mathrm{m}{ }^{\circ} .4\right)$ optical flares.

- The cliromospheric response was greater to U-band (photospheric) flares than to coronal flares (13 Oclober data).

- Most significantly, prolonged X-ray activity can occur with little or no response at other layers of the atmosphere. 
$A$ case has recently been reported of no $X$-ray response at all to a $\Delta U=1^{m} .2$ U-band flare on $Y Z$ CMi (1)oyle et al 1986), perhaps because of the absorption of X-rays by an overlying, dense prominence. llowever, their alternative suggestion, that is, that the event occurred low down in the atmosphere, seems to sugerest the best explanation for the present EV Lac results, namely that the lower levels (photosphere and chromosphicese) are at times magnetically isolated, or largely isolated, from the corona. Supporting evidence for this view is the association, in the EV Lac observations, of a stronger chiromosplieric response to I)-band, rather than X-ray, fares and, of course, the almost complete lack of response in the photosphere and chromasplecre to some 2 hours of X-ray activity on 13 October.

LV Lac is not the only $M$ dwarf to provide evidence for the occasional existence of magnetically isolated regions in the: upper atmospherc; the most spectacular case is the 6 March 1979 X-ray flare on Proxima Cintanri (IIaisch et al, 1981) where there were no related enhancements at all in simultaneous UV, optical or riddio coverage. It should be emphasized that most simultancous observations of cool stars show chromospheric and/or photospheric and/or T'R correlates to an X-ray flare: in these cases magnetic loops apparently permeate the various levels of the star's atmosphere in a manner similar to what is seen on the Sun.

On the Sun, in fact, the existence of magnetically isolated regions in the corona has been demonstrated. Flare-like events with no chromospheric (II cr) counterpart were recorded by the IIXIS experiment on SMIM (3.5 $5.5 \mathrm{ke}: \mathrm{V}$ ) and by the (iOl'S-2 satellite $(0.5-4.0 \mathrm{keV})$ several hours after the November 6, 1980 flare (Svestkit et al 1983). 'These events were also seen in radio observations of the upper coronat but no TR or lower atmosplieric risponse was found. It is proposed that the coronal events originated in a plasmoid that becinne magenetically issolated from the lower atmospheric layers following a major 2-ribbon flare. Tworitbon flares have becol observed on $M$ dwarls (Ilaisch et al. 1983), so it does not seem unreasonable that an anilogous magnetic detachment conld also occur.

\section{ACKNOWIEDCGMENIS:}

CWA is pleised to acknowledge the support of NASA grants NAG5-82 and NGL-06-003-057 to the University of Colorido.

\section{REFERENCES}

Ambruster, C.W., Sciortino, S., and Golub, I. 1987, Ap.J. Supp\}. 65, 273.

Ambruster, C., Suyder, W.A., and Wood, K.S. 1984, A p.J. 284, 270.

Ambruster, C.W., Pettersen, B.R., Hawley, S.L., Coleman, L.A, and

Samdminn, W.II. 1986, in 'New Iusights in Astrophysics', Proc.

Joint NASA/LSA/SERC Conference, University College London, ESA SP-263.

Collura, A., Maggio, A., Sciortino, S., Serio, S., Vaiana, S.S., and

Rosiner, R. 1987, Ap.J. 315, 310.

Doyle, J.G, Butler, C.J., Haisch. M.M., and Rodono, M. 1986, M.N.R.A.S. 223, 1P.

Ilaisch, 13.M., 先 al. 1981, Ap.J. ?2.15, 1009.

Haisch, B.M., Linsky, J.L., Bornmann, P.L., Stencel, R.E., Antiochos,

S.K., Golub, L., and Vaiana, G.S. 1983, Ap.J. 267, 280.

Kodaira, K, Ichinura, K., and Nishimura, S. 1976, Publ. Astron. Soc. Japan $2 \underline{8}, 665$.

Pellersen, B.R., Lvans, D.S., and Colenan, L.A. 1983, Astron. \& Ap. 282, 21.

Riviman, G., and Shevchenko, V.S. 198:, Sov. Astron. Lett. $\underline{8}(2), 85$.

Schmill, J.ll M.MI., Pallivicini, R., Monsignori-Fossi, B.C., and II arnden,

F.R., Jr. 1987, Astrou. \& Ap. 179, 193.

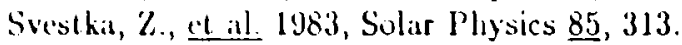


EV LOC, 1985 DAY 288

EV LOC, 1985 DAY 286

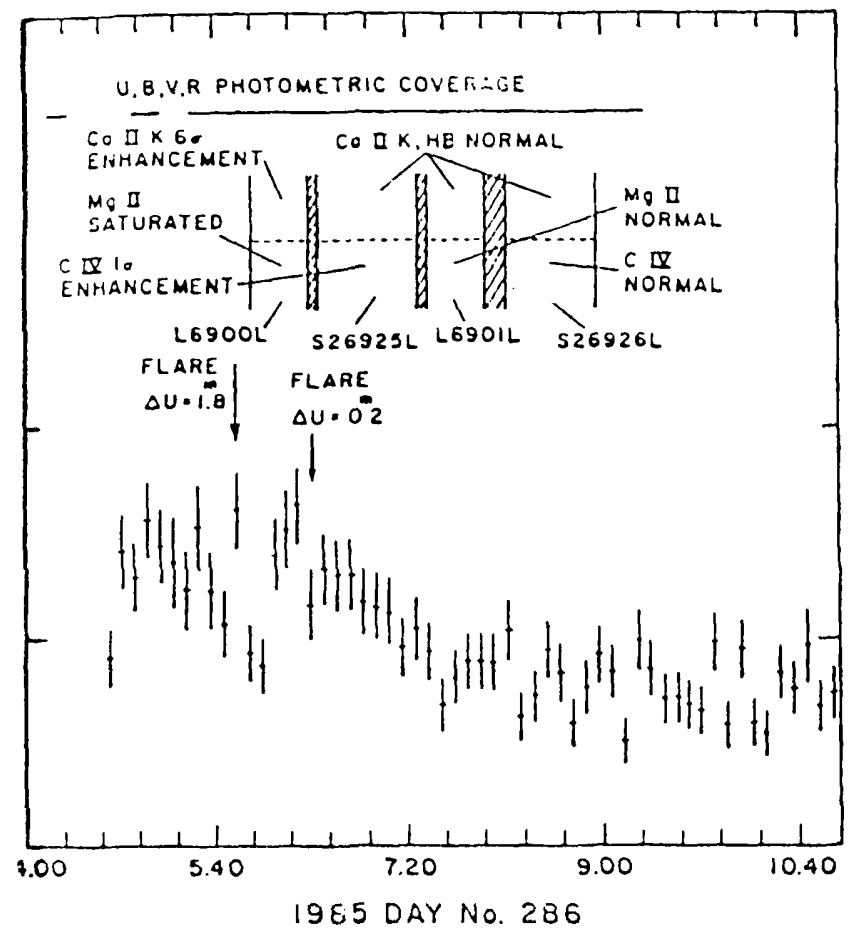

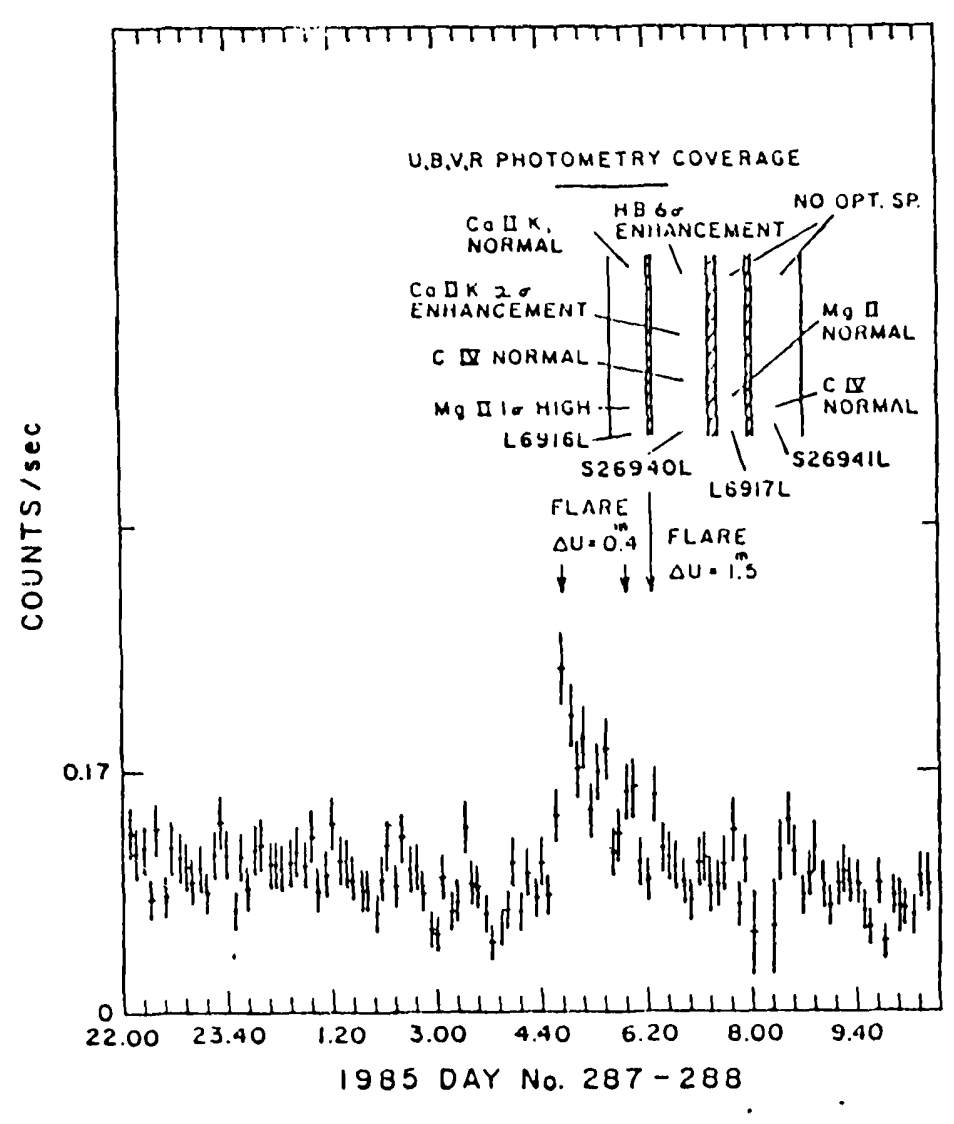

Eiqure 2. EXOSAT LE (0.04-2.0 keV) light curve for EV Lac, 15 oct. 1985. Times of the IUE exposures and other important features are indicated.

gure 1. EXOSAT LE (0.04-2.0 keV) ght curve for EV Lac, 13 oct. 1985. mes of the 4 IUE exposures are dicated between vertical lines, ong with important features at tical and UV wavelengths.

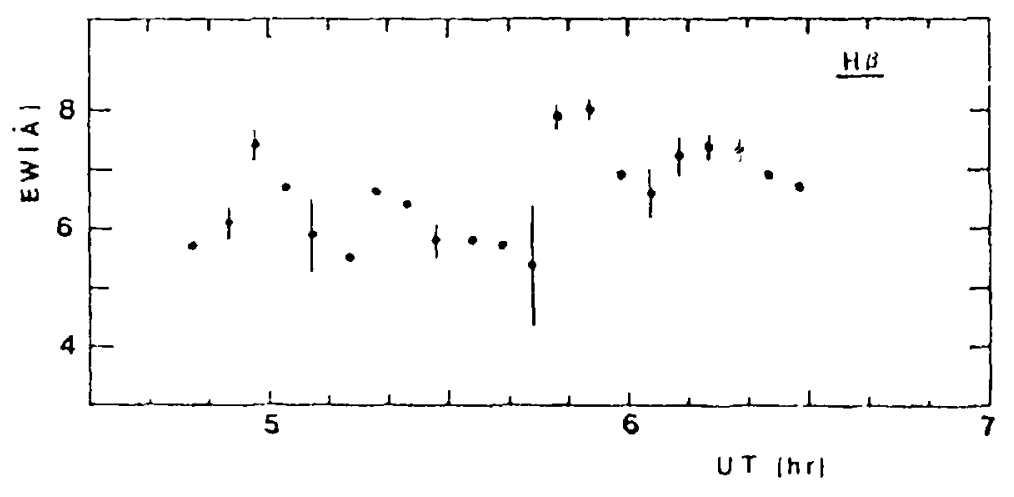
Eigure 3. H-beta light curve for
EV Lac, 13 Oct. 1985. One-sigma error bars are smaller than symbol size for several points.

